\title{
A Study on CO Detection System of Vehicle Emissions based on QEPAS
}

\author{
Zheng Gang, Qi Yingjie* and Li Wei \\ Northeast Forest University, Harbin, 150040, China \\ 11509656@qq.com
}

\begin{abstract}
In this paper, the technology of QEPAS was used to detect the concentration of carbon monoxide gas. The regulation of DBF laser's input current frequency and temperature was made first, current modulation frequency being $f=f_{0} / 2=16420 H Z$ (frequency modulation is half of the resonance frequency of the QTF) and operating temperature being $T=23$ degrees Celsius. Then enable the output wavelength to be near $1.567 \mu \mathrm{m}$ and measure $\mathrm{CO}$ gas of different concentrations. Through the analysis of the relationship between the second harmonic signals of different concentration $\mathrm{CO}$ gas, it is found that between $\mathrm{CO}$ concentration and the second harmonic intensity exists good linear relationship and that the system has no independence on the incident light wavelength and has high sensitivity and large dynamic measurement range. The study provides a new way of thinking for other types of trace gas detection and can be applied to the detection of automobile exhaust emissions.
\end{abstract}

Keywords: Vehicle emissions; QEPAS; Carbon dioxide (CO); Second harmonic.

\section{Introduction}

Exhaust gas or flue gas is emitted as a result of the combustion of fuels such as natural gas, gasoline petrol, biodiesel blends or fuel oil [1] . According to the type of engine, it is discharged into the atmosphere through an exhaust pipe, flue gas stack or propelling nozzle. It often disperses downwind in a pattern called an exhaust plume. It is a major component of motor vehicle emissions (and from stationary internal combustion engines), Crankcase blow-by and Evaporation of unused gasoline can also be included.

A 2013 study by MIT indicates that 53,000 early deaths occur per year in the United States alone because of vehicle emissions [2]. Carbon monoxide poisoning is the most common type of fatal air poisoning in many countries [3] . Carbon monoxide is colorless, odorless and tasteless, but highly toxic. It combines with hemoglobin to produce carboxyhemoglobin, which is ineffective for delivering oxygen to bodily tissues.

Sensor systems that detect and quantify the concentration of specific trace gases will become essential components of urban air quality monitoring, industrial process control [4], and medical diagnostics using breath biomarkers [5-6]. Recently, there has been a growing interest in quartz-enhanced photoacoustic spectroscopy (QEPAS) sensors which use a quartz tuning fork as a resonant acoustic transducer [7-8].

\section{Experimental Setup}

The QEPAS sensor system is shown as Fig 1. The light source is a DFB diode laser with wavelength nearly of $1.567 \mu \mathrm{m}$ and power of $24 \mathrm{~mW}$. One sine signal generating from a function generator (Agilent 33120A) is sent to the DFB laser driving current modulation port, modulated at a half of $f_{0}$ ( $f_{0}$ is QTF resonant frequency) [9-10], and the other sine signal which is a reference signal is sent to the lock-in amplifier simultaneously. The detection light output by the laser passes through two rallying of the 
tuning fork quartz crystal after being focused by the fiber focuser $(1 \mathrm{~cm})$ [11]. The output signal of the quartz tuning fork oscillator was input to the lock-in amplifier after passing through the preamplifier of a $10 \Omega$ electrical Resistance [12-13]. Then the $2 f$ signal output by the lock-in amplifier is collected by DAU, and finally, recorded and displayed by a computer.

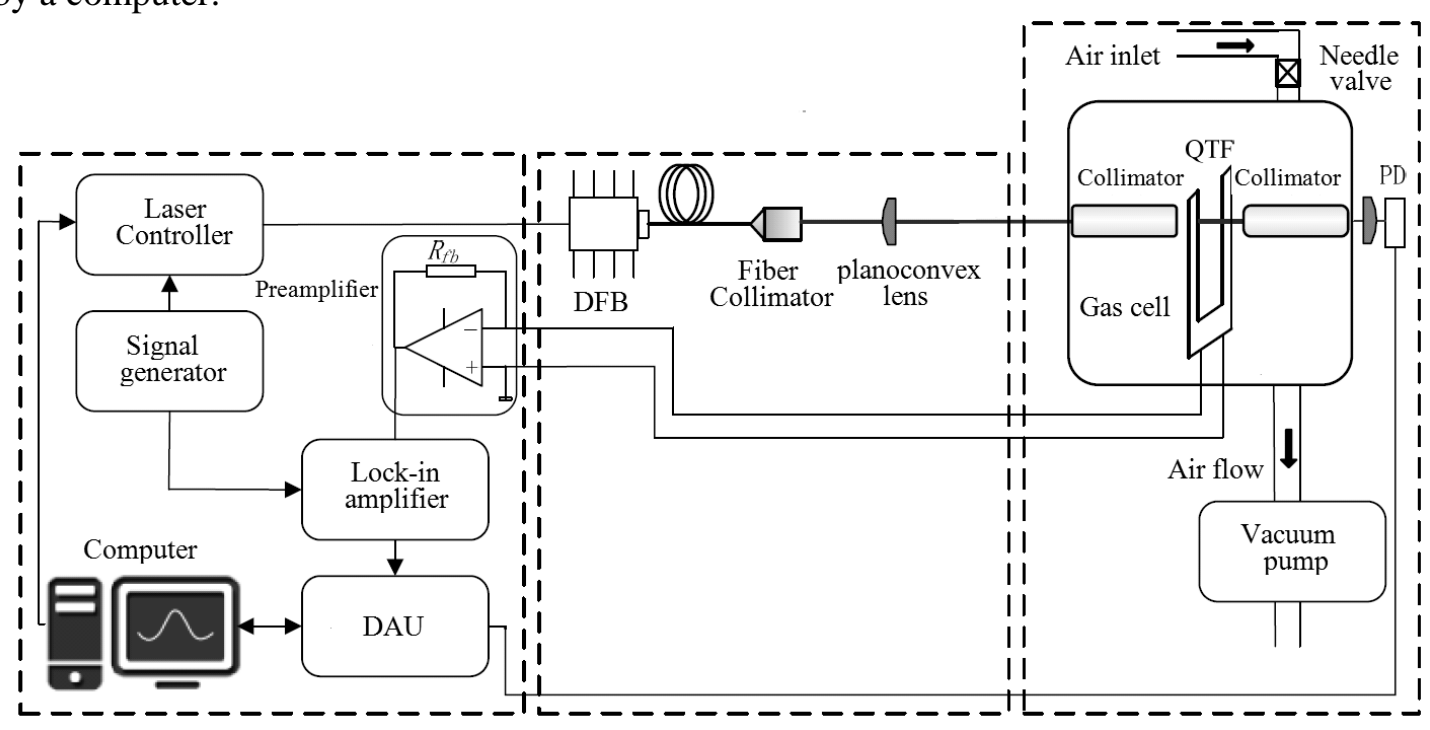

Figure 1. Experimental Setup for the QEPAS System

\section{Results and Discussion}

\subsection{Test of DFB Semiconductor Laser's Operating Characteristics}

Before the QEPAS system is used, the operating characteristics of the laser system should be measured and the modulation system parameters should be optimized. Then use wavelength modulation concentration for the calibration of the system, and test the performance of the QEPAS system, including the detection limit, repeatability, and stability. QEPAS system is used in this study to measure the standard gas of different concentrations and to gain the corresponding two harmonic signals. Then the gas concentration is retrieved by the signal. Wavelength modulation method has the advantages of high sensitivity, high selectivity and being rapidness.

The power and wavelength of Laser changes is the injection of temperature and current changes. In order to determine the relationship between each laser power and wavelength and the temperature and current injection, the temperature value had been fixed when testing and the relationship between the power and the wavelength of the current had been recorded. Temperature value had been fixed at $6{ }^{\circ} \mathrm{C}, 7^{\circ} \mathrm{C}, 8^{\circ} \mathrm{C}, 9^{\circ} \mathrm{C}$ and $10^{\circ} \mathrm{C}$, laser injection current range was from $50 \mathrm{~mA}$ to $130 \mathrm{~mA}$, the laser output wavelength range had been shown in the Fig 2. As can be seen from the figure, with the increase of the injection the laser current, the laser output wavelength can be approximated as a linear increase. 


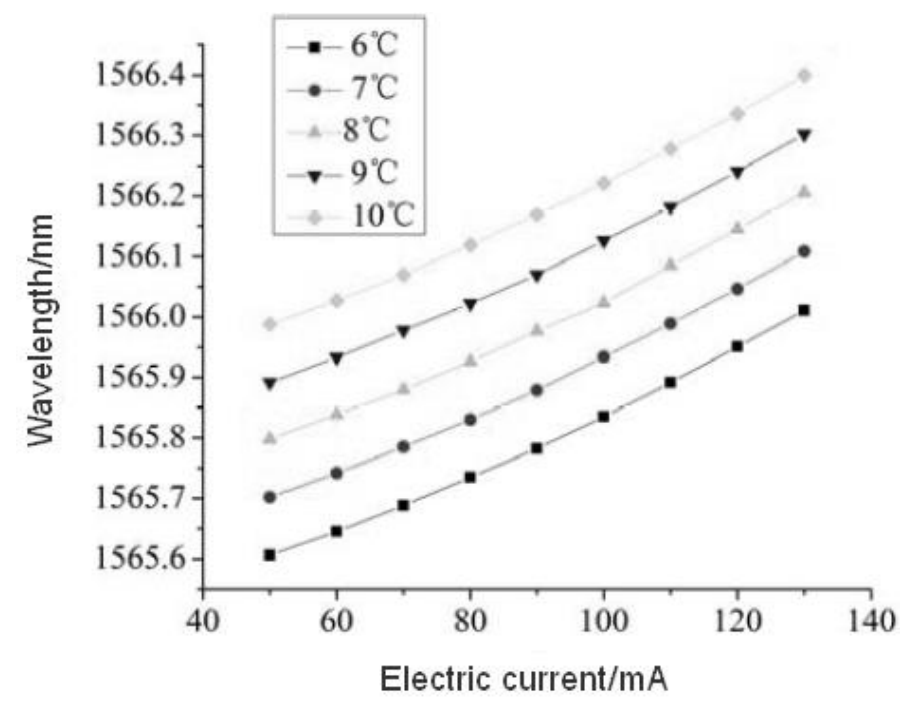

Figure 2. Relationship between Laser Output Wavelength and Current

When the current was in the range of $50 \mathrm{~mA}$ to $130 \mathrm{~mA}$, the laser output wavelength range was $1565.606 \sim 1566.4 \mathrm{~nm}$, the current tuning rate was about $0.005 \mathrm{~nm} / \mathrm{mA}$. When testing laser power's relationship with the current, the temperature had been fixed at $14{ }^{\circ} \mathrm{C}$, $20^{\circ} \mathrm{C}, 26^{\circ} \mathrm{C}, 32{ }^{\circ} \mathrm{C}$ and $38^{\circ} \mathrm{C}$, when the current was in the range of $50 \mathrm{~mA}$ to $130 \mathrm{~mA}$, Relationship curve of output power and current of laser had been shown in the Fig 3 . Similarly, we can see from the figure, with the increase of the injection current of the laser, the output power of the laser could be approximated as a linear increase.

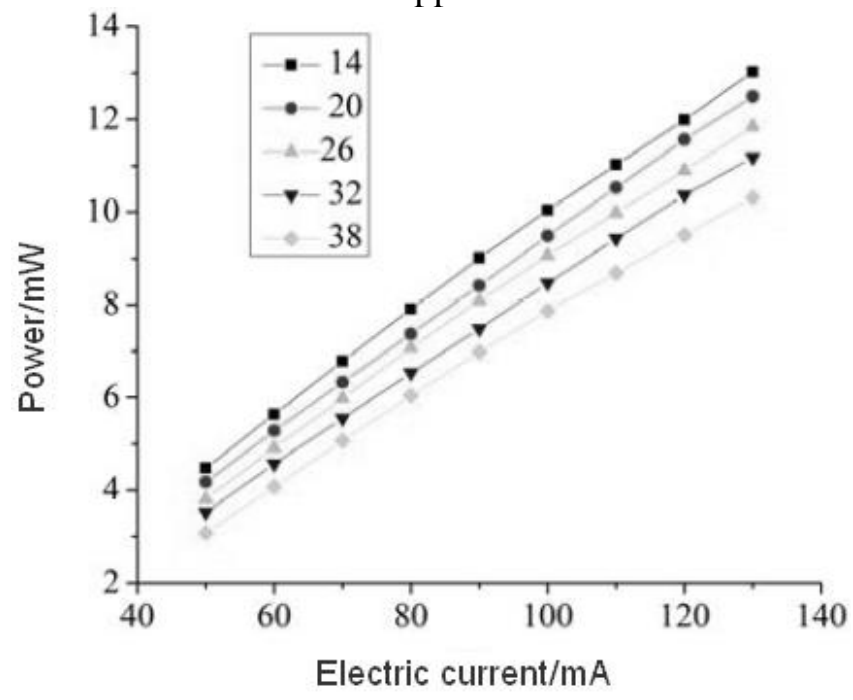

Figure 3. Relationship between Laser output Power and Current

When the relationship between the laser power and the wavelength and the temperature is tested, the current value had been fixed, and the relationship between the power and the wavelength and the temperature of the laser was recorded. Current had been fixed at $50 \mathrm{~mA}, 70 \mathrm{~mA}, 90 \mathrm{~mA}, 110 \mathrm{~mA}$ and $130 \mathrm{~mA}$ and the laser input temperature changes had been controlled within $6 \sim 8{ }^{\circ} \mathrm{C}$, then the curve of laser output wavelength versus temperature was recorded, as shown in the Fig 4 . As can be seen from the figure, with the increase of the temperature of the injection laser, the output wavelength of the laser could be regarded as an approximation of linear increase. The temperature was within the tuning range of $6^{\circ} \mathrm{C}$ to $10^{\circ} \mathrm{C}$, and the temperature tuning rate was about $0.095 \mathrm{~nm} /{ }^{\circ} \mathrm{C}$. 


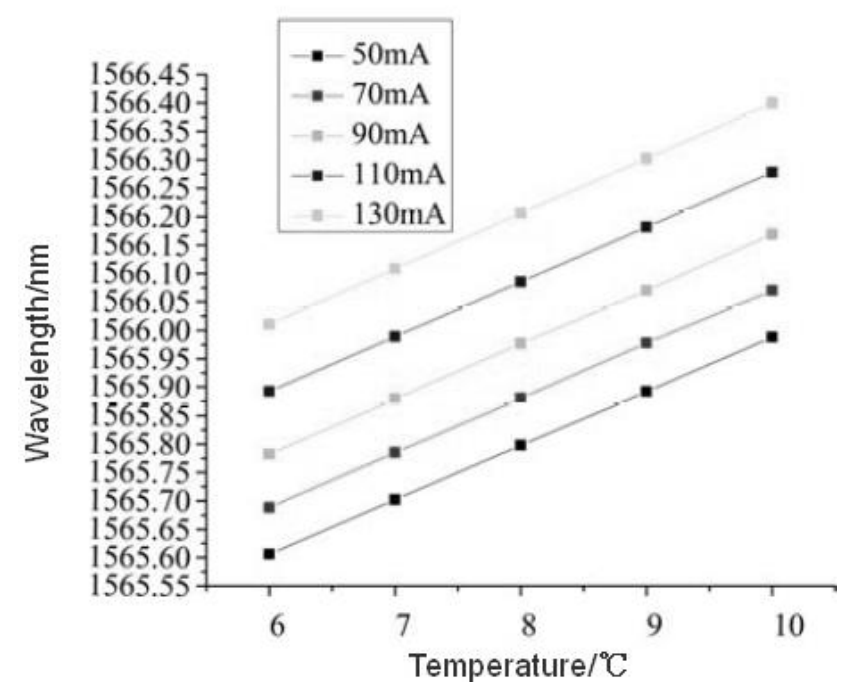

Figure 4. Relationship between Laser Output Wavelength and Temperature

When the relationship between the laser power and the temperature was tested, the current had been fixed in $50 \mathrm{~mA}, 70 \mathrm{~mA}, 90 \mathrm{~mA}, 110 \mathrm{~mA}$ and $130 \mathrm{~mA}$, and the range of the laser temperature control was from $14^{\circ} \mathrm{C}$ to $38^{\circ} \mathrm{C}$, then the curve of the relationship of output power and temperature of laser had been obtained. As shown in the Fig 5. The figure showed that with the increase of temperature of injection laser, the output power of the laser was approximately linear decrease.

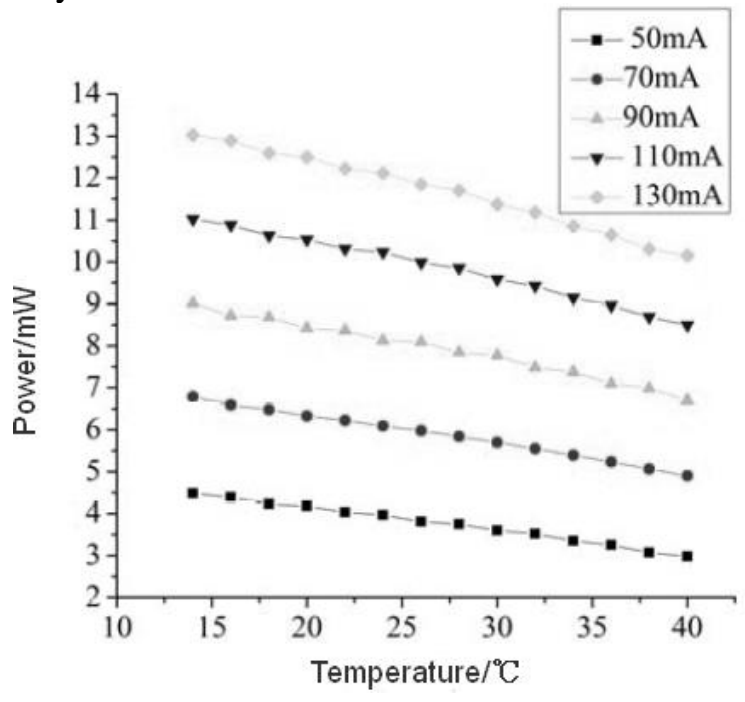

Figure 5. Relationship between Laser Output Power and Temperature

The experimental results had showed that the wavelength of the laser was about $0.005 \mathrm{~nm} / \mathrm{mA}$ and the tuning rate was about $0.095 \mathrm{~nm} /{ }^{\circ} \mathrm{C}$ at DFB. Therefore, when correcting the laser output wavelength, temperature changes was a coarse adjustment and the change of current was a fine adjustment.

\subsection{Modulation Parameters of QEPAS System}

In order to optimize the parameters of wavelength modulation, we need to set and optimize the wavelength modulation parameters in the QEPAS system. By using wavelength modulation method with weak signal detection technology, the sensitivity and 
SNR could be effectively improved. In using the Wavelength modulation method, the choice of Wavelength modulation depth (range) and wavelength modulation frequency affectted the second harmonic of the system, so the selection of processing right parameters had great influence on the detection accuracy of the system.

When wavelength modulation method was used in QEPAS technology, modulation frequency had been set at an optimum frequency that is half of the resonance frequency of quartz tuning fork. In a modulation period, the output wavelength of the laser had passed twice by the center of gas absorption spectrum. When the modulation frequency was half of a tuning fork resonance frequency, we could take the maximum twice harmonic signal. In this paper, the resonant frequency of a quartz tuning fork was $f_{0}=32840 \mathrm{HZ}$, i.e. the modulated frequency of the system was a half of $f_{0}$ and $f=16420 \mathrm{HZ}$.

In order to put the vehicle emissions into the QEPAS system, we need to filter out dust and moisture, the temperature and pressure filtration after the filtering is normal temperature and pressure, and absorption line of the gas to be tested is represented with a Lorentz linear function:

$g_{L}\left(v, v_{0}\right)=\frac{1}{2 \pi} \frac{\Delta v_{L}}{\left(v-v_{0}\right)^{2}+\left(\Delta_{L} / 2\right)^{2}}$,

(1)

Where, $v_{0}$ is the center frequency of the gas to be tested lines, $v$ is the width of the gas absorption line.

Where

$x=v_{c}-v_{0} / \Delta v$,

(2)

$m=a / \Delta v$,

(3)

In this function, $a$ is modulation depth (width), $m$ is modulation factor. Substitute formula (2) and (3) into formula (4), we take

$g(x, m)=1 / 1+(1+m \cos \omega t)$,

(4)

As we know that the harmonic component is proportional to the $\mathrm{N}$ derivative of the absorption coefficient [14-15].

Where

$$
H_{n}\left(v_{c}\right)=\left.\frac{C L}{2^{n-1} n !} \alpha^{n} \frac{d^{n}[\alpha(v)]}{d v^{n}}\right|_{v=v_{c}},
$$

Substitute formula (4) into (5) we can get the signal curve of each harmonic. The second-harmonic is represented with:

$$
H_{2}(x, m)=\frac{4}{m^{2}}-\frac{\sqrt{2}\left(M+1-x^{2}\right) \sqrt{\sqrt{M^{2}+4 x^{2}}+M}+4 x \sqrt{\sqrt{M^{2}+4 x^{2}}}-M}{m^{2} \sqrt{M^{2}+4 x^{2}}} \text {. }
$$

In this Function, $K=1-x^{2}+m^{2}$, in the line center frequency of the gas to be measured, where is $x=0$, get a peak of the second-harmonic signal. Under different modulation factors $m$ which simulated the second-harmonic, we get different theoretical curve of second-harmonic signal. Represented with fig 6.As modulation factor increases, the peak of second-harmonic signal increases first, then decreases and the curve widened at the same time. This may interfere in adjacent lines. In order to avoid interference, we chose the appropriate modulation amplitude to get the best signal of the second harmonic. 


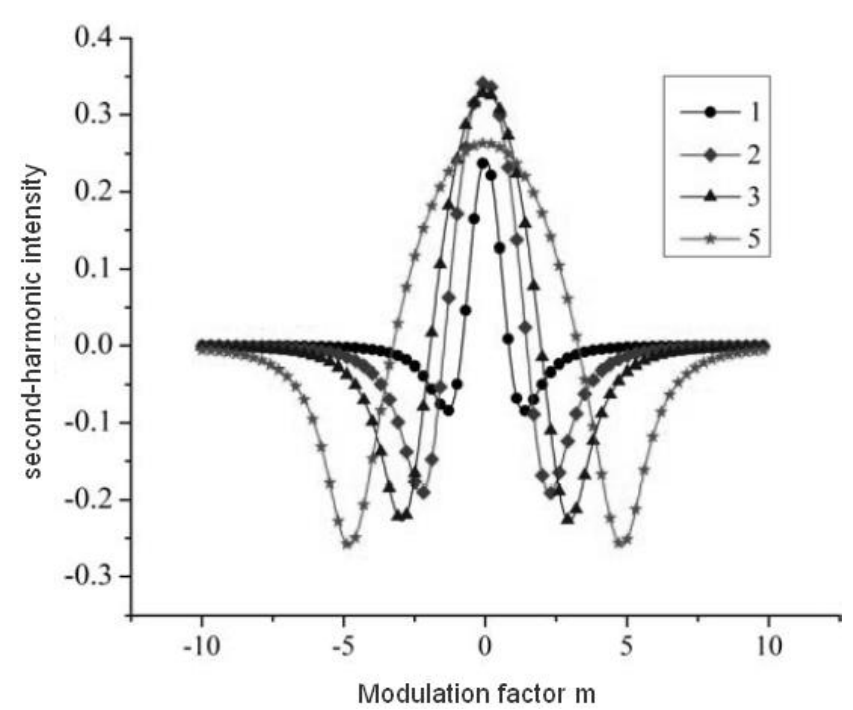

Figure 6. Relationship between Second-harmonic Intensity and Modulation Factor

At the same time, also it got a tuning coefficient and the second harmonic peak graph. Represented with Fig 7. Modulation factor and the second harmonic signal peak was not a linear relationship but achieved a usual maximum of $m=2.2,2.2 \Delta v$ is Optimal modulation depth. $\Delta v$ is the width of the absorption line of the gas to be tested. Gas absorption line width is influenced by pressure. The same kind of gas at different pressures has a different absorption spectrum linewidth, and also a different modulation depth.

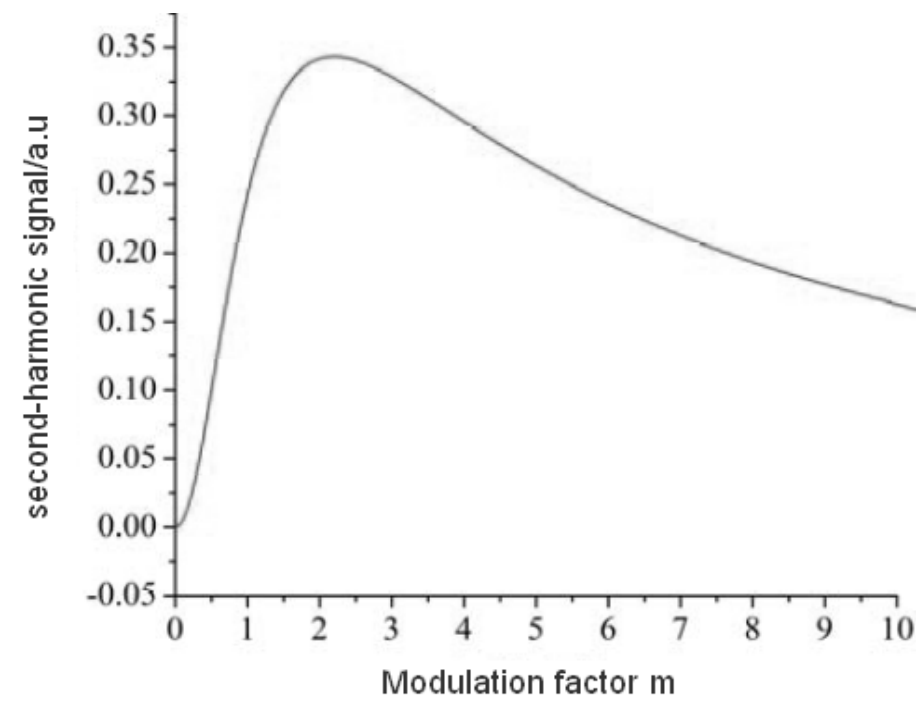

Figure 7. Relationship between Second-harmonic Signal and Modulation Factor

In this paper, measurements were carried out at atmospheric pressure. So we only need to measure the best modulation depth at atmospheric pressure. Measurement was made at modulation depth 1 20mA. The results represent with fig 8 . As is shown, the best modulation depth is $14.1 \mathrm{~mA}$. 


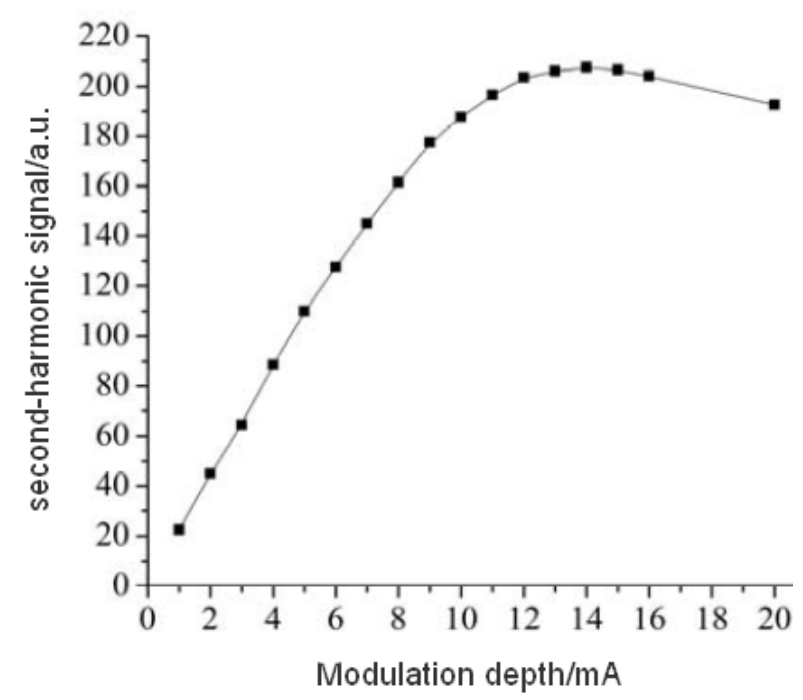

\section{Figure 8. Relationship between Second-harmonic Signal and Modulation Depth}

\subsection{Wavelength Modulation Calibration of QEPAS System}

The auto-exhaust CO Gases detection was used by QEPAS. The method lies in using the measured photoacoustic signal to quantitatively analysize the gas to be tested. After establishing the relationship between the photoacoustic signal and $\mathrm{CO}$ concentrations, the laser power, gas absorption coefficient, quality factor of tuning fork and other system parameters need not be considered. The errors caused by these parameters to calculation then can be avoided, and the concentration of $\mathrm{CO}$ can be inversed directly by using the photoacoustic signal.

Since the second harmonic and the CO concentration peak have the corresponding linear relationship, we can pass the $\mathrm{CO}$ gas of different concentrations of standard into the QEPAS system, and measure the second-harmonic signal peak at different concentrations of CO. After having obtained the linear relationship between second-harmonic peak and concentration of $\mathrm{CO}$, we could use the system to measure the $\mathrm{CO}$ of unknown concentration, and then get the second harmonic of the $\mathrm{CO}$, and this second-harmonic can inverse the concentration of unknown CO.

Before the calibration tests, it was required to continuously pass N2 of high purity into the system for 15 minutes. The aim was purging the system and eliminating the interference from other gases. The electronic control unit (ECU) would control the enter temperature at $23^{\circ} \mathrm{C}$ and the current at $90 \sim 110 \mathrm{~mA}$ to scan the laser during the test in order to adjust and lock the laser output wavelength, and make it scan periodically in the center of CO gas absorption wavelength. Under atmospheric pressure, standard gas of $35 \mathrm{ppm}$. 45ppm 、90ppm 、115ppm and 195ppm was passed into the QEPAS system and photoacoustic detection processed. We had to purge the system of different concentrations prior to gas calibration. The second-harmonic signals of each concentration gas were obtained by calibration, as shown in Fig 9. With the increase of concentration, the secondharmonic peaks (Use arbitrary units (a.u.)) also increase accordingly. 


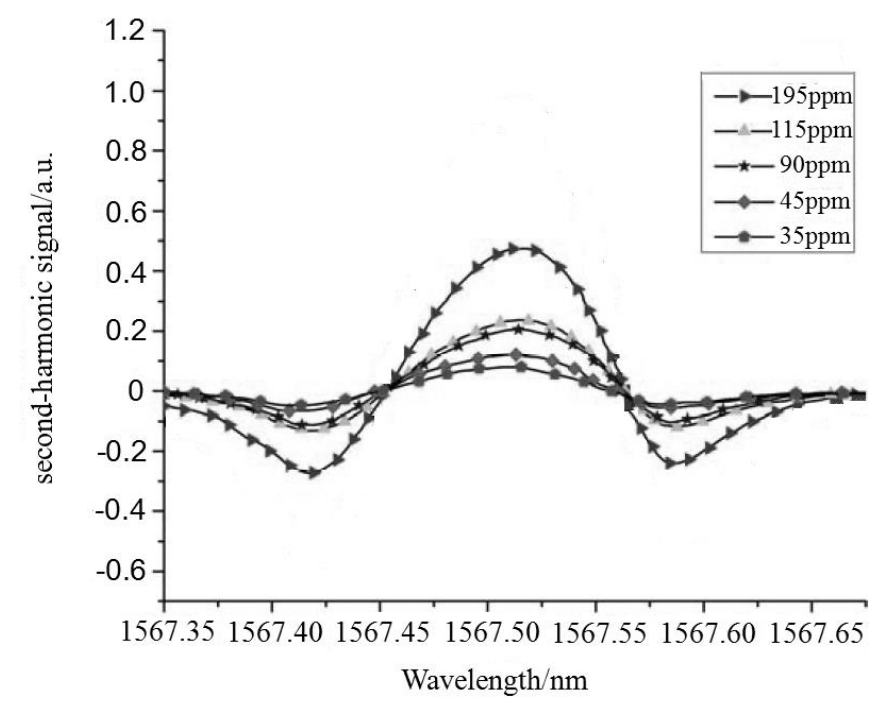

Figure 9. Relationship between Second-harmonic Signal and Wavelength

The information of the second harmonic signal not only depended on the properties of the tested gas absorption, but also was affected by wavelength modulation parameters, thus making the direct using of the second harmonic signal to calculate the concentration of the tested gas more difficult, so we utilizd the second harmonic peak measurement of a standard gas for the concentration inversion. The inversion of $\mathrm{CO}$ gas concentration uses least square method to fit the Relationship between the second harmonic wave signal peaks and concentration.

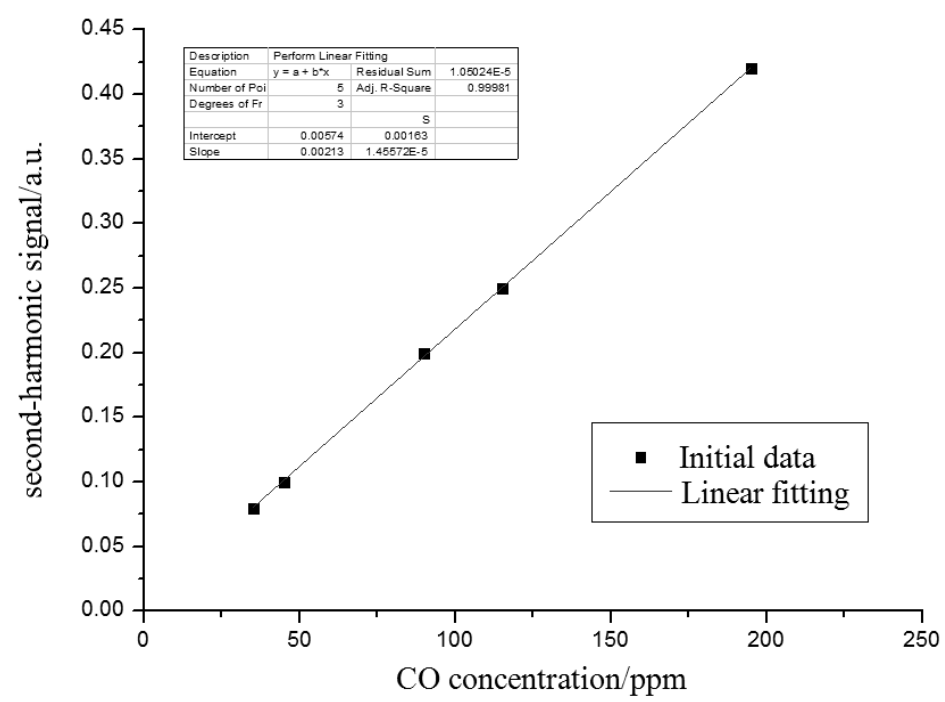

Figure 10. Relationship between the Second-harmonic Signal and $\mathrm{CO}$ Concentration

The least squares formula obtained is $Y=0.00213 X+0.00574$, Fig 10 is a relationship between the second harmonic peak and co concentration. The figure shows that the value of the second harmonic and the concentration of $\mathrm{CO}$ has a good linear relationship within a certain range of concentrations. 


\section{Conclusion}

The carbon monoxide detection system was designed and constructed based on QEPAS technology at the working temperature of 23 degrees Celsius. The two harmonic peaks and the relative concentrations of carbon monoxide were detected by using 35ppm 195ppm.the second-harmonic peak and the relative density of carbon monoxide is linear fitting, This method can be used to effectively measure the concentration of carbon monoxide gas and improve the performance of the detection device of Automobile exhaust.

\section{Acknowledgement}

Qi Yingjie is corresponding author, the paper is supported by a grant from the Program for Twelfth Five-Year National Science and Technology (contracts no: 2013BAJ12B02), the China Communications Construction Company Limited Research\& Development project (contracts no: 2014-ZLKJ-11) and the Fundamental Research Funds for the Central Universities (contracts no: DL12DB03) .

\section{References}

[1] Omidvarborna, "Characterization of particulate matter emitted from transit buses fueled with B20 inidle modes", Journal of Environmental Chemical Engineering, vol. 4, no. 2, (2013), pp. 2335-2342.

[2] Fabio, Ashok and Akshay, "Air pollution and early deaths in the United States. Part I: Quantifying the impact of major sectors", Atmospheric Environment (Elsevier), vol. 79, (2005), pp. 198-208.

[3] G. Yu, B. Dai and J. Chen, "CFD Modeling of a Carbonation Reactor with a K-Based Dry Sorbent for CO2 Capture", Chemical Engineering \& Technology, vol. 37, no. 10, (2014), pp. 1705-1712

[4] A. A Kosterev, G. Wysocki, Y. Bakhirkin, S. So, R. Lewicki, M. Fraser, F. K. Tittel and R. F. Curl, "Application of quantum cascade lasers to trace gas analysis", Appl.Phys, vol. 90, no. 2, (2007), pp. 165-176.

[5] M. R. McCurdy, Y. Bakhirkin, G. Wysocki, R. Lewicki and F. K. Tittel, "Recent advances of laserspectroscopy based techniques for applications in breath analysis", BreathRes, no. 1, (2007), pp. 39-43.

[6] T. H. Risby and S. F. Solga, "Current status of clinical breath analysis", Appl. Phys, vol. 85, no. 2, (2006), pp. 421-426.

[7] A. A. Kosterev, Y. A. Bakhirkin, R. F. Curl and F. K. Tittel, "Quartz-enhanced photoacoustic spectroscopy", Optics Letters, vol. 27, no. 21, (2002), pp. 1902-1904.

[8] T. Laurila, H. Cattaneo, T. Poyhonen, V. Koskinen, J. Kauppinen and R. Hernberg,"Cantilever-based photoacoustic detection of carbon dioxide using a fiber-amplified diode laser", Applied Physics B: Lasers and Optics, vol. 83, no. 2, (2006), pp. 285-293.

[9] J. H.Miller, Y. A. Bakhirkin, T. Ajtai and F. K. Tittel, "Detection OfFormaldehyde Using Off-Axis Integrated Cavity Output Spectroscopy With An Interband Cascade Laser”, Appl. PhysB: Lasers and Optics, vol. 85, no. 4, (2006), pp. 391-396.

[10] K. Melessanaki, V. Papadakis, C. Balas and D. Anglos, "Laser induced breakdown spectroscopy and hyper-spectral imaging analysis of pigments on an illuminated manuscript", Spectrochimica Acta Part B, vol. 56, (2001), pp. 2337-2346.

[11] J. R. Mansfield, M. Attas, C. Mazjels, E. Cloutis, C. Collins and H. H. Mantsch, "Near infrared spectroscopic reflectance imaging:a new tool in art conservation", Vibrational Spectroscopy, no. 28, (2002), pp. 59-66.

[12] Cao, Y., Jin, W., Ho, L. H., and Liu, Z., "Evanescent-wave photoacoustic spectroscopy with optical micro/nanofibers", Opt. Lett, vol. 37, (2012), pp. 214-216.

[13] Schilt S., Kosterev A. A. and Tittel F. K., "Performance evaluation of a near infrared QEPAS based ethylene sensor", Appl. Phys. B: Lasers and Optics, vol. 95, no. 2, (2009), pp. 813-824.

[14] Liu K., Li J., Wang L., Tan T., Zhang W., Gao X., Chen W. and Tittel F. K. "Trace gas sensor based on quartz tuning fork based QEPAS laser photoacoustic spectroscopy", Appl. Phys. B: Lasers and Optics, vol. 94, no. 1, (2009), pp. 527-533.

[15] L. Dong, A. A. Kosterev, D. Thomazy and F. K. Tittel, "QEPAS spectrophones: design, optimization, and performance", Appl. Phys. B: Lasers and Optics, vol. 100, no. 3, (2010), pp. 627-635. 
International Journal of $u-$ and e- Service, Science and Technology Vol.9, No. 2 (2016) 